\title{
Christine de Pizan et Le Livre du Corps de policie
}

\author{
Valérie Toureille, Professeur d'histoire du Moyen Âge, UCP/Paris-Seine
}

Au sein de la littérature politique médiévale, les Miroirs aux Princes forment une catégorie particulière que les littéraires ont abordé bien avant que les historiens ne s'y intéressent ${ }^{1}$. Même si la classification demeure sujette à discussion, comme l'a rappelé Einar Már Jónsson dans un article de $2006^{2}$, les textes regroupés dans cet ensemble répondent à la même finalité : instruire ceux qui exercent le pouvoir, ou qui sont appelés à l'exercer, en vertu du principe selon lequel il faut savoir se gouverner pour bien gouverner. A n'en pas douter, Le Livre du corps de policie de Christine de Pizan, rédigé entre 1406 et 14073, appartient bien à cette catégorie. Destiné au jeune Louis de Guyenne, le troisième fils de Charles vi et d'Isabeau de Bavière, il se présente à la fois comme un manuel d'éducation royale et comme un traité de bon gouvernement. En 1404, le prince a sept ans ; il vient d'être marié avec la fille ainée de Jean sans Peur, duc de Bourgogne, et il est appelé à succéder à un père empêché par la maladie 4 .

L'expression en moyen français - «Mireoir as princes »- est apparue au XIV siècle. Il prolonge le Speculum régale. Le reflet qu'il suggère est celui du modèle. Le prince qui se confronte à son image est invité à la correction ou à l'imitation ${ }^{5}$. Le speculum, à la fois tableau et enseignement, n'est pas cantonné à la politique (c'est-à-dire à la science du bon gouvernement) ; il peut aussi servir à l'enseignement de la religion ou du droit. On en connait les racines et les inflexions. À la fin du XIVe siècle, il prend une

\footnotetext{
${ }^{1}$ Depuis les années 1930, et les travaux fondateurs de Wilhlem Berges et Wilhlem Kleinecke, les miroirs aux princes ont fait l'objet de très nombreux commentaires. Le genre suscite des recherches aussi riches que stimulantes, dont témoignent plusieurs rencontres et tables rondes récentes : Herbert Grabes, Speculum. Miror end Looking Glass, 1973 ; E. Jónsson, Miroir, Naissance d'un genre littéraire, 1995 ; F. Lachaud et L. Scordia (dir.), Le prince au miroir de la littérature politique de l'Antiquité aux Lumières, Rouen, 2007.

2 Einar Már Jónsson, «Les miroirs aux princes sont-ils un genre littéraire ? », Médiévales, 2006, p. 153-166.

${ }^{3}$ Un autre intervalle chronologique est proposé entre 1404 et 1407.

${ }^{4}$ Louis de Guyenne mourra en 1415, au lendemain de la bataille d'Azincourt, à laquelle il n'a pas participé. Le Livre de la Paix, de Christine de Pizan, sera dédicacé au même prince en 1414.

${ }^{5}$ Raymond Darricau, Dictionnaire de la spiritualité, éd. Beauchesne, Paris, 1977, p. 1290 b-1303a et p. $1303 a-1312$ b
} 
tournure nouvelle parce que la monarchie française est en crise et que les institutions commencent à douter, comme un navire sans gouvernail. Une abondante littérature politique va s'épanouir sur ce terrain, celui de l'incertitude. Dans une forme d'urgence, les écrits se multiplient pour rappeler à ceux qui en ont la charge les principes de la juste gouvernance. L'exercice ne manque évidemment pas d'ambiguïté, entre la valorisation du modèle en place et la tentation du sermon, les conseilleurs n'étant pas confrontés eux-mêmes à l'exercice du pouvoir. Le Livre du Corps de policie s'inscrit ainsi dans une tradition ancienne, dont il ne s'écarte pas. Il est aussi, à notre connaissance, le premier traité politique rédigé par une femme, mais ce point ne doit pas être sur-interprété comme ce fut parfois le cas, au détriment du fond.

L'ouvrage de Christine de Pizan se compose de trois parties, annoncées par l'auteur dès son introduction : la première s'adresse aux princes, la deuxième aux chevaliers et aux nobles et la troisième à "l'université de tout le peuple ». Cette tripartition du corps social est conforme à l'image d'un ensemble organique, tel que se le représente les médiévaux. Le roi en est la tête, «le chef » au sens étymologique, les chevaliers et les nobles en sont les bras et les mains. Le peuple en forme le ventre nourricier, les jambes et les pieds. Pour autant, si Christine de Pizan dispense ses conseils aux trois états, l'essentiel de son propos porte sur l'éducation du prince, qui justifie à lui seul trente-trois chapitres, quand les nobles et les chevaliers n'en inspirent que vingt-et-un - dont plusieurs traitent de la "science" militaire ${ }^{6}$ et le peuple seulement onze. L'original subsiste en plusieurs exemplaires. L'un des manuscrits de la Bibliothèque Nationale de France (fr 12439) provient de la bibliothèque de Philippe le Bon. Celui de la Bibliothèque royale de Belgique (10440) est issu, lui aussi, de la collection des ducs de Bourgogne. L'américain Robert-Henri Lucas en a proposé une édition contestée, publiée par Honoré Champion en 1967, en prenant appui sur neuf manuscrits différents. Angus Kennedy, de l'université de Glasgow, l'un des spécialistes de la question, l'a récemment rééditée et corrigée (Honoré Champion, 1998), en s'appuyant sur un manuscrit détenu par la bibliothèque de Chantilly (ms. 294). L'exégèse, elle, s'est surtout intéressée à la langue, aux sources d'inspiration de l'auteur et aux différentes versions du manuscrit, plus qu'à son contenu. Quant au contexte historique de l'oeuvre, il est le plus souvent passé sous silence. La dernière partie de la vie de Christine de Pizan, au cours de laquelle a été écrite Le Livre du corps de policie, correspond à la période des grandes œuvres politiques : la Lamentation sur les maux de la France (1410), le Livre de la paix (1414) ou encore le Dittié de Jeanne d'Arc (1429). De ce point de vue, la connaissance du contexte est essentielle à la compréhension de l'œuvre. En tout état de cause, Le Livre du corps de policie, comme la plupart des autres œuvres de Christine de Pizan, mériterait sans doute une nouvelle édition.

\section{La métaphore du corps politique}

L'usage que fait Christine de Pizan de la métaphore du corps humain comme corps politique est un topos de la pensée politique utilisé depuis l'Antiquité. Platon

\footnotetext{
${ }^{6}$ Christine de Pizan est, par ailleurs, l'auteur du Livre des Faits d'armes et de chevalerie (1410).
} 
l'introduit dans le Timée 7 . Aristote, dans la Politique, compare la cité à un organisme humain. Et la conception de l'État par Sénèque emprunte au même modèle. La pensée médiévale subit par ailleurs l'influence de la tradition ecclésiologique, élaborée à partir de La Cité de Dieu de saint Augustin. La représentation de l'Église comme Corpus Christi, telle qu'on la retrouve sous la plume d'un Jonas d'Orléans au IXe siècle, dans le $D e$ Institutione Regia ${ }^{8}$, conforte cette image, que vient renforcer celle des ordines ou des états du monde, que l'on peut lire sous la plume d'Adalberon de Laon à travers la trifonctionalité des ordres, distinguant « oratores », " bellatores » et «laboratores ». C'est ce modèle qui perdure au XII ${ }^{\mathrm{e}}$ siècle dans les écrits de Guillaume de Normandie et s'épanouit dans le Policraticus de Jean de Salisbury, où il acquiert sa véritable dimension politique ${ }^{9}$. Au corps politique, constitué par la hiérarchie des fonctions, se superpose la notion novatrice de l'État, à la fois entité abstraite et organisation politique. Pour le futur évêque de Chartres, les prêtres sont l'âme du corps social, dont le prince est la tête. Les juges en forment les yeux, les oreilles et la langue, les nobles le corps et les paysans et les artisans les pieds. Cette réflexion se prolonge au XIII siècle avec le livre de Gilles de Rome, traduit au siècle suivant sous le titre de Livre du Gouvernement des princes ${ }^{10}$. Elle est reprise par les théoriciens politiques de la fin du Moyen Âge : Philippe de Mézières ${ }^{11}$ et, surtout, avant lui, Évrart de Trémaugon. Évrard de Trémaugon, que l'on reconnaît aujourd'hui comme l'auteur du Songe du Vergier (écrit entre 1376 et 1378), est considéré comme l'un des plus grands théoriciens politiques de la fin du Moyen Âge. Le Songe appartient à une série d'œ uvres majeures rédigées sous l'impulsion de Charles V, où il côtoie le De Moneta de Nicole Oresme, ses traductions françaises de la Politique, de l'Ethique, de l'Economique, ou bien encore la traduction de la Cité de Dieu réalisée par Raoul de Presles ${ }^{12}$. Le texte de Trémaugon témoigne des préoccupations auxquelles est confronté le roi mais il rend aussi compte des débats qui agitent le cercle de ses

\footnotetext{
${ }^{7}$ La tradition cosmologique issue du Commentaire du Timée par Chalcidius a marqué la pensée médiévale. Le texte était étudié dans les écoles cathédrales.

${ }^{8}$ Jeannine Quillet, Charles V, le roi lettré. Essai sur la pensée politique d'un règne, Paris, Perrin, 1984, p. 211-212.

${ }^{9}$ Jean de Salisbury, Policraticus I-IV, éd. par Katharine Keats-Rohan, Turnhout, Brepols, 1993 ; Frédérique Lachaud, «L'idée de noblesse dans le Policraticus de Jean de Salisbury (1159) », Cahiers de recherches médiévales, 13, 2006, p. 3-19.

10 Gilles de Rome, Le livre du gouvernement des roys et des princes (BNF, lat. 1203), Bell Dora M., L'idéal éthique de la royauté en France au Moyen Âge d'après quelques moralistes de ce temps, Genève, Droz, Paris, Minard, 1962.

${ }^{11}$ Songe du viel pelerin, 1389, éd. Joël Blanchard, avec la collaboration de Antoine Calvet et Didier Kahn, Genève, Droz, 2015, 2 vol. (Textes Littéraires Français, 633) ; trad. par Joël Blanchard, Paris, Pocket, 2008, coll. Agora, $n^{\circ}$ 297). Conseiller à la cour de Charles VI, Philippe de Mézières était proche de Louis d'Orléans.

12 Bertrand Schnerb, "Charles V au miroir du Songe du Vergier», in Le prince en son miroir, Littérature et politique sous les premiers Valois, Actes de la Journée d'études, Dunkerque, 2009, dir. A. Marchandisse, p. 545-559; Janine Quillet, Charles V, op. cit.; Jacques Krynen, L'idéal du prince et pouvoir royal en France à la fin du Moyen Âge (13801440). Étude de la littérature politique du temps, Picard, Paris, 1991 ; Einar Jonson, La naissance, op. cit. ; Frédérique Lachaud et Lydwine Scordia, Le prince au miroir, op. cit. ; B. Ribémont et L. Dulac éd., Une femme de lettres au Moyen Âge. Etudes sur Christine de Pizan, Paradigme, Paris, 2000.
} 
conseillers, «le club du roi » selon l'heureuse formule de Françoise Autrand ${ }^{13}$. Mais le Songe du Vergier n'est pas à proprement parler un miroir aux princes, car l'essentiel de son propos s'articule autour des relations que doivent entretenir le pouvoir temporel et le pouvoir spirituel.

Si Christine de Pizan ne fait jamais référence au Policraticus de Salisbury, pourtant traduit par Denis Foulechat en 1372, elle s'en inspire étroitement, y compris dans la structure du livre ${ }^{14}$. Elle fait d'ailleurs de même dans le Chemin de longue étude ou le Livre de Paix ${ }^{15}$. Elle cite en revanche les Faits et Dits de Maxime Valère, qui viennent d'être traduits, à la demande de Charles V, par Simon de Hesdin et qu'achève Nicole de Gonesse en 1401. Pourquoi insiste-t-elle sur la référence latine ? Sans doute parce que son propos vise à inciter les hommes à la vertu, qu'ils soient prince ou non, et que, sur ce thème, Rome fournit des exemples utiles. Il est d'ailleurs de bon ton, en cette fin du Moyen Âge, de citer les auteurs classiques, comme Sénéque ou Cicéron. Quant au terme "policie », il a été fourni par Nicolas Oresme dans sa traduction d'Aristote. La référence au corps est toujours utilisée, de nos jours, dans les expressions "corps politique » et "corps social», mais c'est sans doute ce dernier terme, contre toute attente, qui prolonge la notion explicitée par Christine de Pizan.

\section{Un moment historique singulier}

L'écriture du livre de Christine de Pizan se situe entre l'achèvement du Livre des faits et bonnes meurs du sage roy Charles $V$, qui magnifie le règne du monarque décédé en 1380, laissant un pays prospère, et l'assassinat de Louis d'Orléans, le 23 novembre 140716. Charles VI est malade depuis 1392. Dans son entourage immédiat, le duc de Bourgogne, son oncle, et le duc d'Orléans, son frère, se vouent une haine mortelle pour savoir lequel des deux tiendra les rênes du pouvoir. La tension est telle que chacun redoute le pire. Tous les espoirs reposent sur l'accession au trône du jeune prince. L'espérance de Christine de Pizan, à cet égard, rejoint celle du royaume.

Le parcours de Christine de Pizan est bien connu, parfois mieux que son œuvre ${ }^{17}$. Son destin épouse les heures et les malheurs de la guerre de Cent Ans, à la charnière entre le XIV et le $\mathrm{XV}^{\mathrm{e}}$ siècle. Née à Venise en 1364 d'un père médecin et astrologue, appelé à Paris l'année suivante auprès du roi Charles $v$, elle découvre la capitale lorsqu'elle est enfant, alors que la situation politique de la France est stabilisée après de longues décennies de troubles. Le roi a commencé à reprendre les territoires concédés aux Anglais et, grâce à l'intelligence militaire de Du Guesclin, il a également jugulé le problème des Grandes Compagnies. Quant au parti Navarrais, il est tenu en échec depuis

\footnotetext{
${ }^{13}$ Françoise Autrand, Christine de Pizan, Fayard, 2009.

14 Voir également la vision du corps social selon Guilaume de Digulleville, Pèlerinage de l'âme, éd. J. J. Sturzinger, Londres, 1893.

${ }^{15}$ Christine de Pizan, Le livre de la paix, éd. Ch. C. Willard, La Haye, 1958.

${ }^{16}$ Christine de Pizan, Livre des fais et bonnes meurs du sage roy Charles Quint, éd. S. Solente, SHF, Paris, 1936

17 Charity C. Willard, Christine de Pizan, her life and works, New York, 1984.
} 
la bataille de Cocherelle (1364). Le médecin italien tisse un solide réseau de fidèles au sein de la cour, qui lui permettra de négocier le mariage de sa fille avec un gentilhomme picard plein d'avenir, notaire et secrétaire du roi : Etienne Castel. Christine de Pizan est mariée à 15 ans, ce qui n'a rien d'exceptionnel au XIVe siècle.

En 1380, alors que la jeune femme a seize ans, la mort de Charles marque un coup d'arrêt à l'accession de la famille Pizan. Puis le décès du médecin laisse sa famille dépourvue. Etienne Castel meurt à son tour, victime d'une épidémie. À vingt-cinq ans, Christine de Pizan se trouve veuve, avec trois enfants à charge, sans compter sa mère et sa nièce. Elle est confrontée à de sérieux problèmes de succession, orchestrés par des hommes de lois qu'elle devait qualifier plus tard « de malotrus et de convoiteux ». Elle ne peut plus compter sur les nouveaux conseillers qui entourent le jeune roi, Charles vi. Son beau-père lui-même a été frappé de disgrâce. Sa situation matérielle se dégrade. Une partie de ses biens est mise sous séquestre ou saisie, tandis qu'elle doit vendre l'autre. Elle décrira plus tard cette transition douloureuse dans la Mutacion de fortune. Christine de Pizan ne voit qu'une issue à son malheur : l'écriture. Elle reprend ses études, ouvre un atelier de copistes, et se lance dans la poésie, en espérant le mécénat des princes.

Lorsque Charles VI, excédé par les querelles de son conseil, rappelle les anciens conseillers de son père - les Marmousets -, l'isolement de Christine de Pizan s'estompe, car ces hommes - Jean Gerson, Gonthier Col, Philippe de Vitry ou Nicolas de Clamanges elle les a tous rencontrés à la cour. Surtout, elle partage leurs convictions. Ils entendent servir le roi et, à travers lui, construire l'État. Ils ont entretenu et développé le premier humanisme français. La définition $\mathrm{du}$ bon gouvernement est au cœur de leur préoccupation. Cette éclaircie est malheureusement de courte durée. En 1392, le jeune Charles VI est frappé par une première crise de folie, qui le laisse hébété pendant plusieurs jours. L'épisode ouvre une longue et douloureuse période de démence intermittente qui devait durer trente années et avoir une importante répercussion dans la vie de Christine de Pizan. La folie du roi était si inattendue qu'on voulut des responsables. On les trouva chez ses conseillers et le peuple de Paris réclama leur exécution. Le roi, revenu à la raison, s'opposa à leur mise à mort, mais la tension demeura grande, incitant plusieurs des accusés à prendre la fuite. Parmi eux, certains apportaient leur soutien à la femme de lettres. La crise ouverte par la maladie du roi élargit ainsi le champ des menaces. En Angleterre, en 1399, Richard II, chassé du trône, est assassiné par Henri IV, le Lancastre. La paix entre la France et l'Angleterre s'en trouve compromise. Les divisions déchirent l'Église. À titre personnel, Christine de Pizan n'est pas épargnée. Elle perd son premier fils dans des circonstances obscures, entre 1396 et 1399. Son autre fils, Jean, placé à la cour d'Angleterre auprès de Richard II, a dû se réfugier en catastrophe auprès du duc de Bourgogne.

Les évènements qui vont se succéder après 1407 confirmeront les craintes de Christine de Pizan. La guerre civile éclate au grand jour après l'assassinat du duc Louis d'Orléans en 1407. Elle perd, à travers lui, un grand mécène. Son fils fuit cette fois la cour de Bourgogne pour rejoindre le camp Armagnac. La suite ne sera qu'une succession de d'épreuves. En 1413, la révolte cabochienne, qui embrase la capitale, se traduit par des horreurs sans nom. En 1415, le massacre d'Azincourt décime les élites du royaume ${ }^{18}$.

18 Elle écrira, à ce sujet L'épistre de la Prison de la vie humaine. 
Louis de Guyenne, sur lequel était fondé tous les espoirs de redressement, meurt la même année. Les Bourguignons investissent Paris en 1418. Christine de Pizan, contrainte de fuir, se réfugie dans le monastère de Poissy, où elle retrouve sa fille, pour y finir ses jours près de dix ans plus tard. Et l'assassinat du duc Jean sans Peur en 1419 annonce le terrible traité de Troyes de 1420. Jamais, dans toute son histoire, la France n'a vécu de moments aussi sombres.

\section{Les vertus du prince idéal}

Le Livre du Corps de Policie n'est pas une œuvre isolée. Il appartient, comme le souligne Angus Kennedy, à un ensemble - une trilogie, plus précisément - qui comprenait également le Livre des fais et bonnes meurs du sage roy Charles $V$ et Le livre de la Paix. Le Livre des fais a été rédigé en 1404, pour répondre à la commande de Philipe le Hardi, duc de Bourgogne, qui voulait louer la personnalité de son frère ainé. Christine de Pizan ne l'a pas conçu comme une biographie, mais comme une réflexion sur le bon gouvernement. En ce sens, le Livre des fais est aussi un miroir au prince. Le Livre de paix, le dernier écrit, date de l'époque de la guerre civile, entre 1412 et 1413 . Dans un plan en trois parties, Christine de Pizan y énumère les sept vertus indispensables à un roi. Ce Livre de la paix, comme le Livre du Corps de policie, est dédié au prince Louis de Guyenne, qui vient d'entrer brillamment sur la scène politique, à l'âge de seize ans, en négociant la paix entre le duc de Berry et le duc de Bourgogne. Le Corps de policie s'intercale donc entre les deux. À eux trois, ils forment une somme théorique, consacrée à la science politique. Leur articulation est d'ailleurs soulignée par l'auteur elle-même en préambule à chacun des trois ouvrages.

Dans le Livre du Corps de policie, Christine de Pizan décline, de manière traditionnelle, les vertus attendues d'un prince idéal. Ce prince doit être semblable au bon berger qui prend soin de protéger son troupeau de la voracité des loups ou la trop grande fréquence des tontes : "Le bon prince qui aimera son pays gardera le sien soigneusement à l'exemple du bon pasteur, si comme il garde ses ouailles, lequel par grande cure pour les défendre des loups et des males beste (c'est ainsi qu'elle designe les soudards) et qu'elles soient nettement tenues et en santé, afin qu'elles puissent accroitre et fructifier et donner laine entière », c'est-à-dire fournir des revenus. Ce berger a besoin de s'appuyer sur des hommes qui présentent les qualités requises, à savoir la sagesse et la loyauté : "Si ordonne (le roi) que iceulx variés soient bien garnis de bons fors chiens à collier ferré". Le bon monarque délègue une partie de son pouvoir à des officiers soucieux du bien public, eux-mêmes protégés par des hommes d'armes loyaux, des " chiens au collier ferré ", capables de s'opposer aux autres hommes de guerre, violents et incontrôlables. La métaphore du berger est bien connue, mais la comparaison entre les chiens et les hommes d'armes est plus hasardeuse. Comme l'indique l'auteur, le chien est courageux, et il n'hésite pas à s'exposer à la mort pour défendre son maître. Surtout, il l'aime "merveilleusement», il lui est d'une loyauté parfaite et il est suffisamment intelligent, pour distinguer ses amis de ses ennemis. N'est-ce pas le portrait idéal du bon "hommes d'armes » gardien du roi, en un temps où la trahison pervertit nombre de relations? 
Mais le prince doit également cultiver des qualités personnelles. Pour être servi sans partage, il doit rester attentif aux requêtes de ses sujets et ne pas écouter les flatteurs. Autrement dit, il ressemble au roi que Christine de Pizan décrit dans le Livre des Fais ${ }^{19}$. Science et prudence font le roi sage. La prudence rappelle l'importance de la diplomatie, en un temps où alliances et contre-alliances varient sans préavis. Par ailleurs, Christine de Pizan, qui se montre intéressée par les questions militaires, n'oublie pas de rappeler que la victoire par les armes continue de définir le roi, tel Charles V, monarque victorieux. À ses yeux, il incarne la sagesse. Si Charles V a gouverné avec ses frères, dans le respect de la tradition, il s'est surtout entouré de théoriciens politiques qui ont contribué à définir la souveraineté du pouvoir royal. Il ne portait pas l'habit court des chevaliers, mais la robe longue des clercs.

Concernant la noblesse, Christine de Pizan insiste sur ses devoirs. Elle n'ignore pas qu'une hiérarchie s'est établie au son sein de cette noblesse, mais, quel que soit leur rang, les nobles doivent vivre dans la crainte de Dieu, le souci du bien public et le respect des institutions, qui sont conformes à la volonté de Dieu. Faute de fréquenter les livres, les nobles doivent s'efforcer de fréquenter les sages. Surtout, ils doivent la loyauté au roi, selon le modèle du chien fidèle.

"L'universalité du commun peuple» - magnifique expression que nous serions fondés à utiliser de nos jours - constitue un ensemble plus hétéroclite, mais une vertu l'unit, qui est l'obéissance. Le commun peuple soutient le corps social, afin qu'il soit «vif, parfait et sain ». Christine de Pizan, qui ne conteste pas l'origine divine du pouvoir des princes, justifie l'obéissance qui leur est due, même lorsque les princes sont injustes, et le paiement à l'impôt, car Jésus lui-même a payé l'impôt de Rome. L'universalité du peuple se partage en trois ordres : Paris et les cités, les bourgeois et marchands, et enfin, en dernier, les artisans et laboureurs. Le premier de ces ordres se confond avec l'état de clergie, en particulier l'Université de Paris et tous les gens de savoir, dont elle fait l'éloge. Viennent ensuite les bourgeois et marchands dont elle exalte le rôle dans la cité, ce qui s'écarte d'une vision traditionnelle du commerce, toujours suspect aux yeux de l'Église. Cette évolution, quant à la perception du marchand, est déjà perceptible chez Nicole Oresme, mais Christine de Pizan attribue à la bourgeoisie un rôle nouveau. Elle peut être utile au gouvernement de la cité, servir de médiateur entre le «menu peuple » et le prince. Le conseil qu'elle donne aux bourgeois est d'endiguer et de contenir les "murmures du peuple», c'est-à-dire la révolte. C'est un phénomène urbain que Christine a éprouvé et redouté, comme beaucoup de Parisiens : les "commotions» urbaines. Ces moments de révolte violente ont en effet jalonné la fin du règne de Charles $\mathrm{V}$ en particulier au sujet de l'impôt, d'où la métaphore des brebis, qui doivent accepter la tonte du berger. Mais Christine de Pizan reconnaît également la fonction économique de la bourgeoisie, indispensable au Corps de policie, car sans elle "l'état des rois et des princes ... ne se pourrait passer ». D'ailleurs, le profit marchand est moins condamnable dès lors qu'il peut aussi se convertir en aumône et en fondations. Le dernier état du peuple se rapporte aux gens de métier et aux laboureurs. Pour ces derniers, Christine n'a que mépris et condescendance : Adam, rappelle-t-elle, chassé du jardin d'Eden, n'était-il pas condamner à labourer la terre? "Nos pères patriarches anciens, ajoute-t-elle, furent tous cultivateurs des terres et pasteurs de bestiaux...et soulait

${ }^{19}$ Janine Quillet, Charles V, op. cit., p. 227. 
mie être anciennement vil office et méprisé». Premier des métiers dans l'ordre de leur apparition, ils sont marqués du sceau de l'infamie originelle. En tant que pieds du corps social, ils sont utiles au gouvernement de la cité mais doivent être étroitement surveillés. Pour le reste, les gens de métiers sont blâmables pour leurs moeurs dégradées voire violentes. Ils sont, il est vrai, le foyer de toutes les révoltes urbaines de la fin du Moyen Âge. Ils sont méprisables pour leur fréquentation des tavernes et leur goût pour les vils plaisirs. Ils sont incultes, et ignorent les Saintes Écritures. Seuls les artisans les plus subtils, tels qu'on en trouve à Paris, trouvent grâce à ses yeux.

Ainsi, Christine de Pizan développe et reprend le thème aristotélicien, cher à la pensée politique médiévale, à travers l'analogie de la santé du corps humain avec la perfection du Corps de policie. Si la moindre partie de ce corps symbolique fait défaut ou entre en division avec l'ensemble, c'en est fait le corps entier qui en souffre : "car tout ainsi que le corps humain n'est mie entier, mais défectueux et déformé quand il lui fault aucun de ses membres, semblablement ne peut le corps de policie être parfait, entier, ni sain. Si tous les états ne sont en bonne conjonction et union ensemble, ainsi qu'ils puissent secourir et aider l'un l'autre, chacun exerçant l'office de quoi il doit servir, lesquels divers offices ne sont, à tout considérer établis, et ne doivent servir [que] pour la conservation de tout ensemble, tout ainsi que les membres de corps humains aident à gouverner et à nourrir tout le corps ». La démonstration est bien connue, d'une certaine manière, Christine de Pizan ressasse une rhétorique un peu usée. Chacun à sa place est comptable de l'ordre établi par Dieu, et il doit se maintenir dans l'état où sa naissance l'a placé : "c'est a savoir, dit-elle, les nobles comme les nobles, les populaires ainsi comme leur appartient, et que tout se réfère à un seul corps d'une même Policie, ensemble vivre en paix et justement, ainsi comme il doit être ». D'une certaine façon, sa vision du corps social est profondément conservatrice et urbaine. Du royaume de France, Christine de Pizan ne connait que Paris, dont elle se méfie à raison, à cause de la versatilité du petit peuple et de sa propension à la violence.

\section{L'éducation du prince}

Dieu ayant donné à l'homme toutes les vertus nécessaires au bon gouvernement, Christine de Pizan les rassemble en bouquet, et les offre à Louis de Guyenne. C'est aussi une femme, une mère qui s'adresse au prince enfant ${ }^{20}$. Face à la folie du roi, et la guerre qui menace, le jeune Louis représente l'espoir et la jeunesse. Le royaume est au bord de la guerre civile et les Anglais menacent de porter à nouveau le feu sur le continent. C'est alors que se pose la question du rôle de l'intellectuel dans la cité : comment servir, comment œuvrer pour le bien commun et recouvrer la paix ? La seule arme que Christine de Pizan maîtrise, dont elle mesure l'impact, est sa plume. Ses écrits sont appréciés à la cour, en particulier par la reine Isabeau de Bavière, à laquelle elle dédie l'un de ses livres, mais également par Jean de Berry et par Louis d'Orléans, tous deux princes de fleur de lys et grands mécènes. Le duc de Bourgogne lui commanda des ouvrages, avant que le paysage politique ne se fracture complètement.

${ }^{20} \mathrm{~F}$. Lachaud et L. Scordia, Au-delà des miroirs, op. cit. évoquent une pédagogie propre à l'enfant, p. 19. 
Christine cherche des explications à la crise politique et des solutions pour guider le futur roi. Elle rappelle que l'équilibre de toute chose réside dans la paix et que chacun est comptable de l'ordre établi par Dieu. Pour cette raison, le devoir du roi est de contraindre chacun à demeurer dans la condition où il est né. L'ordre social dont le roi est le garant n'exige pas l'oppression mais l'unité. C'est au roi d'enseigner leur devoirs aux divers états : "Le bon prince [...] doit vouloir que chacun de ses sujets fasse en paix l'office auquel Dieu l'a établi, les nobles ce qu'ils doivent faire, le clergé entende aux sciences et au service divin, les marchands à leurs marchandises, les gens de métiers à leurs ouvrages, les laboureurs à la culture des terres, et qu'ainsi chacun en son degré vive par bonne policie sans recevoir nulle extorsion, ne charge désordonnée, afin que tous puissent avoir chevance convenable ». Ceux qui perturbent le fonctionnement harmonieux du corps social ce sont les prévaricateurs, les gens de guerre, les larrons ou les fauteurs de guerres civiles.

Son tableau de la société fait directement référence à la situation du moment. Face aux divisions, elle rappelle au roi que les hommes d'armes lui doivent obéissance. Ils doivent abandonner leurs querelles personnelles et renoncer aux guerres privées, pour devenir des "chiens fidèles ». Elle se méfie aussi de la populace, des commotions urbaines, ces désordres qui agitent les villes et qu'elle dénonce dans tous ses livres. L'histoire ne lui donnera pas tort. Elle donne à la bourgeoisie des villes une nouvelle dimension, une nouvelle mission. C'est une alliance ancienne, qui n'est plus dirigée contre les féodaux, mais contre le petit peuple urbain, celui de l'artisanat souvent prompt aux révoltes, en particulier en ces temps de contestation de l'impôt où l'État royal a souvent besoin d'argent.

Dans sa présentation du corps social, Christine de Pizan accorde une part mineure au clergé. Nous sommes loin des trois ordres théorisés par Adalbéron de Laon. Elle range les gens de savoir - ceux de l'Université - dans ce monde des villes. Son propos n'est pas sans rappeler la formation des assemblées représentatives, souvent sollicitées au cours des XIVe et XVe siècles. Le tiers ordre (que l'on appellera quelques décennies plus tard le tiers état) se compose des représentants des bonnes villes (que l'on finit par appeler l'État des bonnes villes). Elle reconnaît ainsi le rôle politique des villes dans le gouvernement du royaume. C'est une innovation sur laquelle il faut insister, peut-être d'inspiration italienne.

Christine de Pizan insiste sur la part essentielle des officiers intègres dans l'équilibre du bon gouvernement, ceux qu'elle a cotoyés (les Marmousets). Elle insistera d'ailleurs sur leur recrutement. Ces officiers ont pour charge de "défendre le droit du prince et de la chose publique». Cette idée est partagée par le mouvement de "reformacion du royaume ». L'État a besoin de subsides, mais il a aussi besoin d'hommes qui le servent loyalement ${ }^{21}$. L'on retrouve ici plusieurs des requêtes des assemblées représentatives (depuis 1358), à savoir le contrôle de l'usage de l'impôt et la nomination

${ }^{21}$ Chez Guillaume de Digulleville, ce qui prime dans sa description du corps social ce sont justement les officiers, les serviteurs de la chose publique, cf Philippe Contamine, «Le Moyen Âge occidental a-t-il connu des serviteurs de l'État», Les serviteurs de l'État au Moyen Âge, Publications de la Sorbonne, Paris, 1999, p. 9-20. 
d'officiers intègres. Elle rappelle l'enjeu de la délégation des pouvoirs, qui fait écho au rôle central de ces assemblées d'Etats. C'est un élément essentiel de la vie politique de son temps, qui explique le modèle des états dans les traités politiques), mais c'est aussi un élément essentiel pour le courant réformiste, en vertu du principe «Quod Omnet tanguit ». Le rôle majeur qu'elle reconnaît aux officiers royaux (un milieu qu'elle connaît bien) : ils forment la véritable colonne vertébrale de l'Etat. Ces hommes doivent porter l'idéal de bien commun et le respect de la chose public. On pourrait dire qu'ils portent une certaine conscience du service public: c'est ce que l'on trouve déjà sous la plume d'un certain nombre de juges et d'officiers, je pense en particulier aux grands baillis.

Enfin, Christine de Pizan rappelle que le roi est un homme mortel, comme les autres, et qu'il n'emportera rien dans l'Autre monde que ses péchés. L'exercice du pouvoir royal est d'essence divine, mais le monarque ne l'est pas. Il appartient à l'humanité. Son office est transitoire, comme l'office de seigneurie. C'est une conception moderne de la souveraineté qu'elle exprime ici. La théorie des deux corps du roi est en gestation. Nous sommes presque au terme d'un processus : le roi n'est que le dépositaire d'un office, même s'il est encore, dans l'esprit des médiévaux, un peu plus que cela.

000

Quand Christine de Pizan écrit le Livre du Corps de Policie, son œuvre, entamée en 1393, comprend déjà plus d'une vingtaine d'opus, dont la célèbre Cité des Dames. La poésie lui a d'abord permis d'attirer l'attention de la reine et celle des princes. Puis elle a commencé à réfléchir à des sujets que nous qualifierions aujourd'hui "de société ». Lorsqu'elle dédie Le Livre du Corps de policie au jeune prince Louis de Guyenne, elle ignore que la situation du royaume va encore s'aggraver. La mort du prétendant au trône viendra ruiner ses espérances, mais le pire reste à venir. En 1410, il ne s'agit plus de conseiller, mais de constater. Les lamentations sur les maux de la France ouvrent un nouveau cycle. Dix années plus tard, le funeste traité de Troyes (1420) livre la couronne de France au roi anglais. Ce n'est plus la personne du roi qui constitue le cœur de ses préoccupations, dès lors qu'il est incapable de défendre son "troupeau», mais une entité abstraite que la guerre a rassemblé dans le malheur. La "grant pitié du royaume de France » démembre l'image d'un corps articulé. Toute harmonie a disparu. Une nouvelle littérature apparaît qui exalte un sentiment jusque-là inconnu : le patriotisme. Dans ce contexte politique marqué par une profonde inquiétude, la défense de la Nation et l'exaltation de la monarchie alimentent les traités ${ }^{22}$. Pendant près de dix ans, la femme de lettres ne pourra plus écrire, jusqu'à l'apparition d'une jeune femme de Lorraine qui offre au pays un nouvel espoir, à laquelle elle consacre, au seuil de sa mort, un ultime Dittié de Jeanne d'Arc, consacré à la Pucelle. L'auteur d'une poésie de cour est plus que jamais une femme engagée.

Christine de Pizan doit être lue comme un auteur de son temps. Son œuvre a beaucoup souffert des anachronismes. Utilisée pendant l'occupation pour exalter la cause de la France, étudiée plus récemment comme l'une des figures du militantisme

22 Introduction F. Lachaud et L. Scordia, Au-delà des miroirs, op. cit., p. 9. 
féministe, elle reste malheureusement ignorée pour la partie centrale de son œuvre politique. 